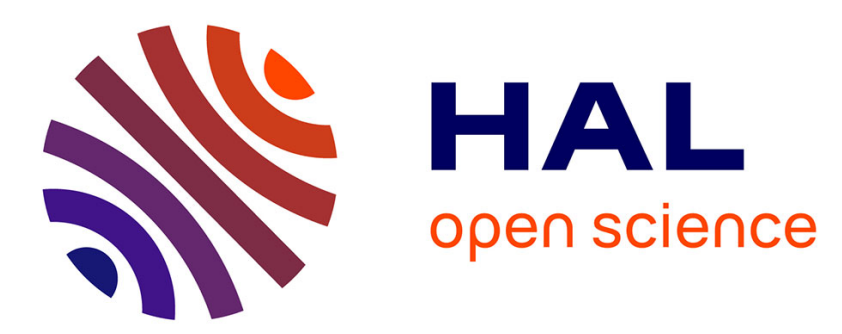

\title{
Necessary and sufficient condition for stabilizability of discrete-time linear switched systems: a set-theory approach
}

\author{
Mirko Fiacchini, Marc Jungers
}

\section{- To cite this version:}

Mirko Fiacchini, Marc Jungers. Necessary and sufficient condition for stabilizability of discretetime linear switched systems: a set-theory approach. Automatica, 2014, 50 (1), pp.75-83. 10.1016/j.automatica.2013.09.038 . hal-00875424

\section{HAL Id: hal-00875424 https://hal.science/hal-00875424}

Submitted on 22 Jan 2014

HAL is a multi-disciplinary open access archive for the deposit and dissemination of scientific research documents, whether they are published or not. The documents may come from teaching and research institutions in France or abroad, or from public or private research centers.
L'archive ouverte pluridisciplinaire HAL, est destinée au dépôt et à la diffusion de documents scientifiques de niveau recherche, publiés ou non, émanant des établissements d'enseignement et de recherche français ou étrangers, des laboratoires publics ou privés. 


\title{
Necessary and sufficient condition for stabilizability of discrete-time linear switched systems: a set-theory approach *
}

\author{
Mirko Fiacchini ${ }^{\text {a }}$, Marc Jungers b,c \\ ${ }^{a}$ Gipsa-lab, Grenoble Campus, 11 rue des Mathématiques, BP 46, 38402 Saint Martin d'Héres Cedex, France \\ (email: mirko.fiacchini@gipsa-lab.fr) \\ ${ }^{\mathrm{b}}$ Université de Lorraine, CRAN, UMR 7039, 2 avenue de la forêt de Haye, Vandouvre-lès-Nancy Cedex, 54516, France \\ (email: marc.jungers@univ-lorraine.fr)
}

${ }^{\mathrm{c}} \mathrm{CNRS}, \mathrm{CRAN}$, UMR 7039, France

\begin{abstract}
In this paper, the stabilizability of discrete-time linear switched systems is considered. Several sufficient conditions for stabilizability are proposed in the literature, but no necessary and sufficient. The main contributions are the necessary and sufficient conditions for stabilizability based on set-theory and the characterization of a universal class of Lyapunov functions. An algorithm for computing the Lyapunov functions and a procedure to design the stabilizing switching control law are provided, based on such conditions. Moreover a sufficient condition for non-stabilizability for switched system is presented. Several academic examples are given to illustrate the efficiency of the proposed results. In particular, a Lyapunov function is obtained for a system for which the Lyapunov-Metzler condition for stabilizability does not hold.
\end{abstract}

Key words: Switched linear systems; set-theory; stabilizability; invariance.

\section{Introduction}

Switched systems are systems for which the current timevarying dynamics, specified by the so-called switching law, belongs to a finite set of modes (see [18]). In the last decades, a large literature has been devoted to study switched systems for practical reasons, as they model complex systems like embedded ones, and for theoretical ones, since their behavior and stability properties are not intuitive nor trivial, [19]. Depending on the assumptions on the switching law, different frameworks have risen. The switching law is often considered as a perturbation or as a part of the control inputs.

When the switching law is a perturbation, that is an arbitrary function, sufficient and conservative conditions to ensure the stability have been provided (see for overviews [22, 23, 27]). Several refinements have been proposed to obtain necessary and sufficient conditions for stability of switched systems. Among these conditions, one can cite the joint spectral radius approach $[1,21,14]$; the polyhedral Lyapunov functions [24] and the path-dependent switched Lyapunov ones [17]. Specific necessary and sufficient conditions exist

\footnotetext{
ऋ Corresponding author M. Fiacchini.
}

for two-dimensional systems [8] or positive ones [13].

In case the switching law is a part of the control inputs, sufficient conditions for stabilizability have been provided, mainly by using a min-switching policy [18, Chapter 3] introduced in [28], developed in [16] via BMI and leading to Lyapunov-Metzler inequalities [11]. Based on the setinduced Lyapunov functions introduced in [4], sufficient conditions for uniform ultimate boundedness have been proposed for uncertain switched linear systems in [20]. Nevertheless to the best knowledge of the authors, necessary and sufficient conditions for the stabilizability and for the nonstabilizability of discrete-time switched linear systems do not exist in the literature.

The proposed approach is based on set-theory and invariance for control and analysis. A seminal work dealing with the characterization of invariance is [2]. More recently, the works $[12,15,5]$ lay the basis of set-theoretic methods and invariance in control. The relation between contractivity and polyhedral Lyapunov functions is presented in [3]. In [24, 4], it is proved that the existence of a contractive polytope is a necessary and sufficient condition for exponential stability and that polyhedral functions form a universal class of Lyapunov functions for linear parametric uncertain systems. 
See [6] for a detailed review on the topic.

This paper provides new necessary and sufficient conditions for stabilizability and sufficient ones for non-stabilizability of discrete-time linear switched systems. A family of nonconvex, homogeneous functions is proved to be a universal class of Lyapunov functions for switched linear systems. The key idea is to use the set-theory offering numerically sound algorithms to check the stabilizability (and providing the stabilizing switching law) or the non-stabilizability. The paper extends the results presented in the preliminary work [9].

The outline of the paper is as follows. In Section 2, preliminaries and set-theory tools are presented. Section 3 is devoted to the new necessary and sufficient conditions for stabilizability. Section 4 provides the stabilizing switching control law. Section 5 presents sufficient conditions for nonstabilizability. Links with the Lyapunov-Metzler approach are discussed in Section 6. The results are illustrated by examples in Section 7, before concluding remarks in Section 8.

Notation: Define $\mathbb{N}_{n}=\{x \in \mathbb{N}: 1 \leq x \leq n\}$ with $n \in \mathbb{N}$. Given $D, E \subseteq \mathbb{R}^{n}, \alpha \in \mathbb{R}$ and $M \in \mathbb{R}^{m \times n}$, define $D+E=$ $\left\{z=x+y \in \mathbb{R}^{n}: x \in D, y \in E\right\}$, the scalar multiple $\alpha D=$ $\left\{\alpha x \in \mathbb{R}^{n}: x \in D\right\}$ and $M D=\left\{M x \in \mathbb{R}^{m}: x \in D\right\}$. Given a set $D \subseteq \mathbb{R}^{n}, \operatorname{int}(D)$ is its interior and $\partial D$ its boundary. The set $\mathbb{B}^{n}$ is the unit euclidean ball in $\mathbb{R}^{n}$. The $i$-th element of a finite set of matrices is denoted as $A_{i}$, of a set of sets as $\Omega^{i}$.

\section{Preliminaries}

Consider the discrete-time switched system

$$
x_{k+1}=A_{\sigma(k)} x_{k}
$$

where $x_{k} \in \mathbb{R}^{n}$ is the state at time $k \in \mathbb{N}$ and $\sigma: \mathbb{N} \rightarrow \mathbb{N}_{q}$ is the switching law that, at any instant, selects the transition matrix among the finite set $\left\{A_{i}\right\}_{i \in \mathbb{N}_{q}}$, with $A_{i} \in \mathbb{R}^{n \times n}$ for all $i \in \mathbb{N}_{q}$. Given the initial state $x_{0}$ and a switching law $\sigma(\cdot)$, we denote with $x_{N}^{\sigma}\left(x_{0}\right)$ the state of the system (1) at time $N$ starting from $x_{0}$ by applying the switching law $\sigma(\cdot)$. In some cases $\sigma$ can be a function of the state, for instance in the case of switching control law, as shown later.

Remark 1 Two main cases have to be discriminated depending on the assumptions on the switching law. If $\sigma(\cdot)$ is supposed to be an arbitrary function of time, that is acting as a perturbation, then the problem of asymptotic stability of the system under every possible switching law is usually considered. For this case necessary and sufficient conditions for stability exist. When $\sigma(\cdot)$ is considered as a manipulable signal, then the problem of asymptotic stabilizability (simply denoted as stabilizability in what follows) is addressed, that consists in the existence and the characterization of the switching laws that yield asymptotic stability if applied. This is the problem considered in the paper.
A concept widely employed in the context of set-theory and invariance is the $\mathrm{C}$-set, see $[4,6]$. A C-set is a compact, convex set with $0 \in \operatorname{int}(\Omega)$. We define an analogous concept useful for our purpose. For this, we first recall that a set $\Omega$ is a star-convex set if there exists $x^{0} \in \Omega$ such that every convex combination of $x$ and $x^{0}$ belongs to $\Omega$ for every $x \in \Omega$.

Definition 1 A set $\Omega \subseteq \mathbb{R}^{n}$ is a $C^{*}$-set if it is compact, starconvex with respect to the origin and $0 \in \operatorname{int}(\Omega)$.

We define the analogous of the gauge function of a $C^{*}$-set as

$$
\Psi_{\Omega}(x)=\min _{\alpha \geq 0}\{\alpha \in \mathbb{R}: x \in \alpha \Omega\}
$$

for the $C^{*}$-set $\Omega \subseteq \mathbb{R}^{n}$. In what follows, we will refer to $\Psi_{\Omega}(x)$ as the Minkowski function of $\Omega$ at $x$, with a slight abuse since it is usually defined for C-sets (or symmetric C-sets), [25, 26, 6]. Some basic properties of the $\mathrm{C}^{*}$-sets and their Minkowski functions are listed below. The proof is avoided, since they follow directly from the definition.

Property 1 Any $C$-set is a $C^{*}$-set. Given a $C^{*}$-set $\Omega \subseteq \mathbb{R}^{n}$, we have that $\alpha \Omega \subseteq \Omega$ for all $\alpha \in[0,1]$, and the Minkowski function $\Psi_{\Omega}(\cdot)$ is: homogeneous of degree one, i.e. $\Psi_{\Omega}(\alpha x)=\alpha \Psi_{\Omega}(x)$ for all $\alpha \geq 0$ and $x \in \mathbb{R}^{n}$; positive definite; defined on $\mathbb{R}^{n}$ and radially unbounded.

The Minkowski functions induced by $\mathrm{C}$-sets have been used in literature as Lyapunov functions candidates, see [3]. In particular, it has been proved that they provide a universal class of Lyapunov functions for linear parametric uncertain systems, [24, 4], and switched systems with arbitrary switching, [22]. In this paper we prove that the Minkowski functions induced by $\mathrm{C}^{*}$-sets form a universal class of Lyapunov function for switched systems with switching control law. For this, we provide a definition of Lyapunov function for the particular context, in analogy with the definition given in [4] for linear parametric uncertain systems.

Definition 2 A positive definite continuous function $V: \mathbb{R}^{n} \rightarrow \mathbb{R}$ is a global Lyapunov function for the system (1) if there exist a positive $N \in \mathbb{N}$ and a switching law $\sigma(\cdot)$, defined on $\mathbb{R}^{n}$, such that $V$ is non-increasing along the trajectories $x_{k}^{\sigma}(x)$ and decreasing after $N$ steps, i.e. $V\left(x_{1}^{\sigma}(x)\right) \leq V(x)$ and $V\left(x_{N}^{\sigma}(x)\right)<V(x)$, for all $x \in \mathbb{R}^{n}$.

The Definition 2 is a standard definition of global Lyapunov function (or, better, global control Lyapunov function) except for the $N$-steps decreasing requirement. On the other hand, such a function implies the convergence of every subsequence in $j \in \mathbb{N}$ of the trajectory, i.e. $x_{i+j N}^{\sigma}(x)$ for all $i<N$, then also the convergence of the trajectory itself. This, with the stability assured by $V\left(x_{1}^{\sigma}(x)\right) \leq V(x)$, ensures global asymptotic stabilizability of the switched system. 


\section{Stabilizability of switched systems}

It is proved in [24] that for an autonomous linear switched system, the origin is asymptotically stable if and only if there exists a polyhedral Lyapunov function, see also [4, 22]. Our main objective is to prove that analogous results can be stated in the case that the switching sequence is a properly chosen selection, that is considering it as a control law.

The system (1) is asymptotically stabilizable if there exists a switching law and a Lyapunov function for the resulting time-varying system. The switching law will belong to the class of state-dependent one, that is $\sigma(k)=g\left(x_{k}\right)$, where $g: \mathbb{R}^{n} \rightarrow \mathbb{N}_{q}$. We define, with a slight abuse of notation, the state-dependent switching law as $\sigma(k)=\sigma\left(x_{k}\right)$.

Assumption 1 The matrices $A_{i}$, with $i \in \mathbb{N}_{q}$, are nonsingular.

Remark 2 Assumption 1 is not restrictive. In fact, the stable eigenvalues of the matrices $A_{i}$ are beneficial from the stability point of view of the switched systems and poles in zero are related to the most contractive dynamics. Moreover, the results presented in the following can be extended to the general case with appropriate considerations. Finally, recall that sampled linear systems do not present poles in the origin and then real systems satisfy Assumption 1.

Consider the following algorithm:

Algorithm 1 Computation of a contractive $C^{*}$-set for the system (1) satisfying Assumption 1.

- Initialization: given the $C^{*}$-set $\Omega \subseteq \mathbb{R}^{n}$, define $\Omega_{0}=\Omega$ and $k=0$;

- Iteration for $k \geq 0$ :

$$
\begin{aligned}
& \Omega_{k+1}^{i}=A_{i}^{-1} \Omega_{k}, \quad \forall i \in \mathbb{N}_{q}, \\
& \Omega_{k+1}=\bigcup_{i \in \mathbb{N}_{q}} \Omega_{k+1}^{i} ;
\end{aligned}
$$

- Stop if $\Omega \subseteq \operatorname{int}\left(\bigcup_{j \in \mathbb{N}_{k+1}} \Omega_{j}\right)$; denote $\check{N}=k+1$ and

$$
\check{\Omega}=\bigcup_{j \in \mathbb{N}_{\check{N}}} \Omega_{j} .
$$

From the geometrical point of view, $\Omega_{k+1}^{i}$ is the set of $x$ mapped in $\Omega_{k}$ through $A_{i}$. Then $\Omega_{k+1}$ are the $x \in \mathbb{R}^{n}$ for which there exists a selection $i(x) \in \mathbb{N}_{q}$ such that $A_{i(x)} x \in \Omega_{k}$. Thus, $\Omega_{k}$ is the set of $x$ that can be driven in $\Omega$ in at most $k$ steps and hence $\check{\Omega}$ the set of those which can reach $\Omega$ in $\check{N}$ or less steps, by an adequate switching law.

Proposition 1 The sets $\Omega_{k}^{i}$ and $\Omega_{k}$ with $i \in \mathbb{N}_{q}$ and for all $k \geq 0$ are $C^{*}$-sets.
Proof: Clearly $\Omega_{0}$ is a $C^{*}$-set. It is sufficient to prove that $A^{-1} D$ and $D \cup E$ are $C^{*}$-sets, for all nonsingular $A \in$ $\mathbb{R}^{n \times n}$ and every $C^{*}$-sets $D$ and $E$ to prove the results by induction. By definition $\alpha x \in D$ for all $x \in D$ and $\alpha \in[0,1]$. Then given $\alpha \in(0,1]$ we have

$$
\begin{aligned}
\alpha A^{-1} D & =\left\{\alpha x \in \mathbb{R}^{n}: A x \in D\right\}=\left\{y \in \mathbb{R}^{n}: A y \in \alpha D\right\} \\
& \subseteq\left\{x \in \mathbb{R}^{n}: A x \in D\right\}=A^{-1} D,
\end{aligned}
$$

since $D$ is a $C^{*}$-set. For $\alpha=0, \alpha A^{-1} D=\{0\} \subseteq A^{-1} D$, trivially. Then $A^{-1} D$ is a star-convex set and it is also compact from Assumption 1. It contains the origin in its interior since $A_{i}^{-1}$ are continuous operators under Assumption 1. Then $A^{-1} D$ is a $C^{*}$-set. The property on the union follows from the definition of $\mathrm{C}^{*}$-set.

Algorithm 1 provides a $\mathrm{C}^{*}$-set $\check{\Omega}$ contractive in $\check{N}$ steps, for every initial $C^{*}$-set $\Omega \in \mathbb{R}^{n}$, if and only if the switched system (1) is stabilizable. Such a necessary and sufficient condition, which is the main contribution of the paper, is stated in the theorem below.

Theorem 1 There exists a Lyapunov function for the switched system (1) if and only if Algorithm 1 ends with finite $\check{N}$.

Proof: Sufficiency follows from the fact that, if the algorithm ends in finite time, then $\check{\Omega}$ induces a Lyapunov function. Indeed, $\check{\Omega}$ being a $C^{*}$-set from Proposition 1 , its Minkowski function is defined. Moreover, considering

$$
\check{\lambda}=\check{\lambda}(\Omega)=\min _{\lambda}\{\lambda \geq 0: \Omega \subseteq \lambda \check{\Omega}\},
$$

we have that $\check{\lambda}<1$, since $\Omega \subseteq \operatorname{int}(\check{\Omega})$ and $\check{\Omega}$ is a $C^{*}$-set. Since $\check{\Omega}$ is the set of $x$ such that $x_{k}^{\sigma}(x)$ are in $\Omega$ for $k=k(x) \leq \check{N}$, and for an appropriate switching sequence, then

$$
x_{k(x)}^{\sigma}(x) \in \Omega \subseteq \check{\lambda} \check{\Omega},
$$

for all $x \in \check{\Omega}$ and in particular for $x \in \partial \check{\Omega}$. This means that there exists a switching $\sigma(x)$ and $k(x) \leq \check{N}$ such that

$$
\Psi_{\check{\Omega}}\left(x_{k(x)}^{\sigma}(x)\right) \leq \check{\lambda} \Psi_{\check{\Omega}}(x),
$$

for all $x \in \partial \check{\Omega}$. Then the Minkowski function decreases after $k(x)$ step, for all $x$ on the boundary. Moreover, it does not increase, for all $j \leq k(x)$. In fact, given $x \in \partial \check{\Omega}$, the elements $x_{j}^{\sigma}(x)$ can be stirred in $\Omega$ in $k(x)-j$ steps for all $j \leq k(x)$, being elements of the same sequence whose last element is in $\Omega$. This means that $x_{j}^{\sigma}(x) \in \check{\Omega}$ and then

$$
\Psi_{\check{\Omega}}\left(x_{j}^{\sigma}(x)\right) \leq \Psi_{\check{\Omega}}(x), \quad \forall j \in \mathbb{N}_{k(x)},
$$


for all $x \in \partial \check{\Omega}$. Then for every $x \in \partial \check{\Omega}$ there exists a switching sequence of length $k(x)$ such that the $\Psi_{\check{\Omega}}$ is not increasing for the first $k(x)-1$ steps and it decreases of at least a proportional value $\check{\lambda}$ at the instant $k(x)$, from (7) and (8). Since every $x$ is on the boundary of a level set of $\Psi_{\check{\Omega}}(x)$, in particular $x \in \partial\left(\Psi_{\check{\Omega}}(x) \Omega\right)$, and from the homogeneity of the Minkowski function and the linearity of the switched system, we have that (7) and (8) hold for every $x \in \mathbb{R}^{n}$. Thus from (7) and (8) valid on the whole $\mathbb{R}^{n}$, we have

$$
\Psi_{\check{\Omega}}\left(x_{\check{N}}^{\sigma}(x)\right) \leq \check{\lambda} \Psi_{\check{\Omega}}(x),
$$

for all $x \in \mathbb{R}^{n}$ which proves that $\Psi_{\breve{\Omega}}$ is a Lyapunov function. Then, if Algorithm 1 ends with finite $\check{N}$, a Lyapunov function exists, in particular $\Psi_{\check{\Omega}}(x)$.

To prove necessity, suppose that there exists a Lyapunov function for the switched system (1). We demonstrate that this implies finite termination of Algorithm 1. From Definition 2 , there is a switching law $\sigma(\cdot)$, a positive value $N \in \mathbb{N}$ and a continuous positive definite function $V: \mathbb{R}^{n} \rightarrow \mathbb{R}$ such that for every $x$ we have $V\left(x_{N}^{\sigma}(x)\right)<V(x)$. Consider the set

$$
\Omega^{V}=\left\{x \in \mathbb{R}^{n}: V(x) \leq 1\right\},
$$

which is closed from continuity of $V$ and bounded from its radially unboundedness. Hence $\Omega^{V}$ is compact and $0 \in$ $\operatorname{int}\left(\Omega^{V}\right)$, since $V$ is continuous and positive definite. Thus for every $\mathrm{C}^{*}$-set $\Gamma$, there exists $\varepsilon>0$ such that the $\mathrm{C}^{*}$-set $\varepsilon \Gamma$ satisfies $\varepsilon \Gamma \in \operatorname{int}\left(\Omega^{V}\right)$. Posing $\Omega=\varepsilon \Gamma$ in Algorithm 1, we have $\Omega \subseteq \operatorname{int}\left(\Omega^{V}\right)$. From the globally asymptotic stability of the system (1), there exists a switching law $\sigma(x)$ defined on $\mathbb{R}^{n}$ and a finite $N_{V} \in \mathbb{N}$ such that for all $x \in \Omega^{V}$ there exists $k(x) \leq N_{V}$ for which $x_{k(x)}^{\sigma}(x) \in \Omega$. Consider $\Omega_{N_{V}}$ obtained by applying Algorithm 1 with $\Omega$ defined above, supposing that the stop condition has not been satisfied, otherwise the result would be directly proved. Since the set $\Omega_{j}$ is the set of states that can be stirred in $\Omega$ in at most $j$ steps, then $\Omega^{V} \subseteq$ $\bigcup_{j \in \mathbb{N}_{N_{V}}} \Omega_{j}$ and then we have $\Omega \subseteq \operatorname{int}\left(\Omega^{V}\right) \subseteq \operatorname{int}\left(\bigcup_{j \in \mathbb{N}_{N_{V}}} \Omega_{j}\right)$, that contradicts the fact that the stop condition has not be satisfied. Then Algorithm 1 terminates with $\check{N} \leq N_{V}$.

Then finite termination of Algorithm 1 is a necessary and sufficient condition for the global asymptotic stabilizability of the switched system (1). An alternative formulation of such a necessary and sufficient condition is presented below.

Theorem 2 There exists a Lyapunov function for the switched system (1) if and only if there exists a $C^{*}$-set whose Minkowski function is a Lyapunov function for the system.

Proof: The result follows directly from the proof of Theorem 1, where the Minkowski function of $\check{\Omega}$ is proved to be a Lyapunov function for the switched systems.

Theorem 2 states the main theoretical contribution of the paper, namely that the existence of a Lyapunov function induced by a $\mathrm{C}^{*}$-set is a necessary and sufficient condition for stabilizability of switched systems. Hence, such functions, nonconvex and homogeneous of degree one, form a class of universal Lyapunov functions for the switched systems. Such result is in line with the fact that the existence of convex Lyapunov functions is sufficient but not necessary for the stabilizability of a switched system, as proved in [7].

Remark 3 The Algorithm 1 terminates after a finite number of iterations only if the switched system is stabilizable, then there is no guarantee of finite termination in general (which means it is a semi-algorithm, to be exact, as noticed by a reviewer). In Section 5 below, a sufficient condition for non-stabilizability is provided that could be used to exclude the existence of a Lyapunov function and then to terminate the (semi-)algorithm. Nevertheless, there are cases in which stabilizability cannot be assessed nor excluded by means of a proper algorithm, i.e. after a finite number of iterations. This is not surprising as in the nonlinear context the problem of the existence a Lyapunov function is often undecidable. In our case, we proved that a Lyapunov function exists and is computable if the switched system is stabilizable.

\subsection{Duality robustness-control of switched systems}

In this section, we recall some results from the literature on the stability of a switched linear system with arbitrary switching law $\sigma(\cdot)$. Our purpose is to highlight the evident analogies with the approach proposed in this paper concerning stabilizability of switched linear systems.

Consider the linear switched system (1) and assume that the switching law is arbitrary. This would mean that the switching law might be regarded as a parametric uncertainty and the results in $[24,3,4]$ on robust stability apply with minor adaptations, see also [22]. The following algorithm provides a polytopic contractive set, and then an induced polyhedral Lyapunov function, for this class of systems, see [6].

Algorithm 2 Computation of a $\lambda$-contractive $C$-set for the system (1) with arbitrary switching law.

- Initialization: given the $C$-set $\Gamma \subseteq \mathbb{R}^{n}$ and $\lambda \in[0,1)$, define $\Gamma_{0}=\Gamma$ and $k=0$;

- Iteration for $k \geq 0$ :

$$
\begin{aligned}
& \Gamma_{k+1}^{i}=\lambda A_{i}^{-1} \Gamma_{k}, \quad \forall i \in \mathbb{N}_{q}, \\
& \Gamma_{k+1}=\Gamma \cap \bigcap_{i \in \mathbb{N}_{q}} \Gamma_{k+1}^{i} ;
\end{aligned}
$$

- Stop if $\Gamma_{k} \subseteq \Gamma_{k+1}$; denote $\hat{N}=k$ and $\hat{\Gamma}=\Gamma_{k}$.

The set $\hat{\Gamma}$ is the maximal $\lambda$-contractive set in $\Gamma$ for the switched system with arbitrary switching law. Provided the Algorithm 2 terminates with finite $\hat{N}$, it can be proved that the system is globally exponentially stable, see [4].

Remark 4 Notice the analogies between the Algorithms 1 and 2: they share the same iterative structure and they both 
generate contractive sets which induce Lyapunov functions provided they terminate in a finite number of steps. The main substantial difference consists in the use of intersection/union operators and in the family of sets generated, $C^{*}$-sets by Algorithm 1 and $C$-sets by Algorithm 2. Interestingly, the $C$-sets are closed under the intersection operation whereas $C^{*}$-sets are closed under the union. The $C^{*}$-sets have for switched systems with switching control law the role that $C$-sets have for the case of arbitrary switching law.

We finally recall that, for linear parametric uncertain systems, the existence of a polyhedral Lyapunov function is a necessary and sufficient condition for asymptotic stability.

Theorem 3 ([24, 4]) There exists a Lyapunov function for a linear parametric uncertain system if and only if there exists a polyhedral Lyapunov function for the system.

The result in Theorem 3 holds for general parametric uncertainty and applies also for switched systems with arbitrary switching law, as remarked in [22].

Remark 5 As for the duality of the Algorithms 1 and 2 highlighted in Remark 4, evident conceptual analogies hold between Theorem 2 and Theorem 3. Then the class of Minkowski functions induced by $C^{*}$-sets is universal for linear switched systems with switching control law, in analogy with the class of polyhedral functions (i.e. induced by C-sets) for the case of arbitrary switching law, [3, 4].

\section{Switching control law}

Besides a Lyapunov function, Algorithm 1 provides a stabilizing switching control law or, better, a family of stabilizing control laws for system (1), if it terminates in finite time.

Proposition 2 If Algorithm 1 ends with finite $\check{N}$ then $\Psi_{\check{\Omega}}$ : $\mathbb{R}^{n} \rightarrow \mathbb{R}$ is a Lyapunov function for the switched system (1) and given the set valued map

$$
\check{\Sigma}(x)=\arg \min _{(i, k)}\left\{\Psi_{\Omega_{k}^{i}}(x): i \in \mathbb{N}_{q}, k \in \mathbb{N}_{\check{N}}\right\} \subseteq \mathbb{N}_{q} \times \mathbb{N}_{\check{N}},
$$

any switching law defined as

$$
(\check{\sigma}(x), \breve{k}(x)) \in \check{\Sigma}(x),
$$

is a stabilizing switching law and such that

$$
\begin{aligned}
& \Psi_{\check{\Omega}}\left(x_{\check{k}(x)}^{\check{\sigma}}(x)\right) \leq \check{\lambda} \Psi_{\check{\Omega}}(x), \\
& \Psi_{\check{\Omega}}\left(x_{j}^{\check{\sigma}}(x)\right) \leq \Psi_{\check{\Omega}}(x), \quad \forall j \in \mathbb{N}_{\check{k}(x)},
\end{aligned}
$$

with $\check{\lambda}$ as in (5).

Proof: The fact that $\Psi_{\check{\Omega}}(\cdot)$ is a Lyapunov function has been proved in the proof of Theorem 1. Denote $\alpha=$
$\Psi_{\check{\Omega}}(x)$, to simplify the notation. Then $x \in \partial(\alpha \check{\Omega})$ by definition. Moreover, from definition of $\check{\Omega}$, there are some values $(i, k) \in \mathbb{N}_{q} \times \mathbb{N}_{\check{N}}$ such that $x \in \partial\left(\alpha \Omega_{k}^{i}\right)$, since $\check{\Omega}$ is the union of $\Omega_{k}^{i}$ for all $i \in \mathbb{N}_{q}$ and $k \in \mathbb{N}_{\check{N}}$. Concerning the $(i, k)$ for which $x \in \partial\left(\alpha \Omega_{k}^{i}\right)$ is not satisfied, we have that $x \notin\left(\alpha \Omega_{k}^{i}\right)$ and then $\Psi_{\Omega_{k}^{i}}(x)>\alpha$. This is due to the fact that $x$ is either on the boundary or in the complement of every $\alpha \Omega_{k}^{i}$, for all $i \in \mathbb{N}_{q}$ and $k \in \mathbb{N}_{\check{N}}$, otherwise $\alpha$ would not be the minimal value such that $x \in \alpha \Omega$. Then for every $i \in \mathbb{N}_{q}$ and $k \in \mathbb{N}_{\check{N}}$ we have two possibilities: either $\Psi_{\Omega_{k}^{i}}(x)=\alpha$, if $x \in \partial\left(\alpha \Omega_{k}^{i}\right)$, or $\Psi_{\Omega_{k}^{i}}(x)>\alpha$, if $x \notin\left(\alpha \Omega_{k}^{i}\right)$. By construction, $\Omega_{k}^{i}$ is the set that can be stirred in $\Omega$, and then also in the contracted set $\check{\lambda} \check{\Omega}$, in $k$ steps by means of a sequence of modes whose first element is $i$. Moreover, the Minkowski function does not increase along the first $k-1$ elements of the generated trajectory, see the proof of Theorem 1. Then from homogeneity of the Minkowski functions, the set $\Sigma(x)$ is composed by the $(i, k)$ where $i$ is the first element of a control sequence $\sigma(x)$ that leads to have $x_{k}^{\sigma}(x) \in \check{\lambda} \alpha \check{\Omega}$ and $x_{j}^{\sigma}(x) \in \alpha \check{\Omega}$ for all $j \in \mathbb{N}_{k}$. As $(\check{\sigma}(x), \breve{k}(x))$ is a selection of the set $\Sigma(x)$, the result follows.

It could be reasonable, to speed up the convergence of the trajectory of the system to origin, to select among the elements of $\Sigma(x)$, those whose $k$ is minimal.

Corollary 1 If Algorithm 1 ends with finite $\check{N}$ then the switching law defined by (12) and (13) is such that

$$
\Psi_{\check{\Omega}}\left(x_{p \check{N}}^{\check{\sigma}}(x)\right) \leq \check{\lambda}^{p} \Psi_{\check{\Omega}}(x),
$$

for every $p \in \mathbb{N}$ and all $x \in \mathbb{R}^{n}$.

Proof: From Proposition 2 we have that, if Algorithm 1 ends with finite $\check{N}$, then there exists a switching law $\check{\sigma}(x)$ and the related $\breve{k}(x) \leq \breve{N}$ such that the Minkowski function of $\check{\Omega}$ does not increase for $k \leq \breve{k}(x)$ and it decreases of a proportional value of $\check{\lambda}$ after $\breve{k}(x)$ steps, for all $x \in \mathbb{R}^{n}$. Since $\breve{k}(x) \leq \check{N}$, then the value of $\Psi_{\check{\Omega}}(x)$ decreases at least once within the next $\check{N}$ steps, that means that

$$
\Psi_{\check{\Omega}}\left(x_{\check{N}}^{\check{\sigma}}(x)\right) \leq \check{\lambda} \Psi_{\check{\Omega}}(x)
$$

that implies (14) as the property applies on the whole $\mathbb{R}^{n}$.

Remark 6 If the system is asymptotically stabilizable, then the algorithm ends with finite $N$ for all initial $C^{*}$-set $\Omega$. Clearly, the value of $\check{N}$ and the complexity of the set $\Omega$ depend on the choice of $\Omega$. In particular, if $\Omega$ is the euclidean norm ball (or the union of ellipsoids), the sets $\Omega_{k}^{i}$ and $\Omega_{k}$, with $i \in \mathbb{N}_{q}$ and $k \in \mathbb{N}_{\check{N}}$, are unions of ellipsoids, and so is $\check{\Omega}$. Then, the switching law computation reduces to check the minimal value among $x^{T} P_{j} x$ with $j \in \check{M}$, where $\left\{P_{j}\right\}_{j \in \check{M}}$ are the $\check{M}$ positive definite matrices that define $\check{\Omega}$, with $\check{M}=$ 
$q+\cdots+q^{\check{N}}=\left(q^{\check{N}+1}-q\right) /(q-1)$, for $q>1$ and $\check{M}=\check{N}$ for $q=1$. Moreover, if $\Omega$ is a polytope (or a union of polytopes), also $\Omega_{k}^{i}, \Omega_{k}$, with $i \in \mathbb{N}_{q}$ and $k \in \mathbb{N}_{\check{N}}$, and $\check{\Omega}$ are so. In this case, the switching law is obtained by evaluating the linear inequalities defining those polytopes.

\section{Non-stabilizable switched system}

In this section, conditions ensuring that there is not a switching law such that the system (1) converges to the origin are given. We will refer to this case as non-stabilizability of the switched system. First, a sufficient condition is provided.

Proposition 3 Given the switched system (1) and the $C^{*}$-set $\Omega \subseteq \mathbb{R}^{n}$ and denoting

$$
\bar{\Omega}=\bigcup_{i \in \mathbb{N}_{q}} A_{i}^{-1} \Omega
$$

if $\bar{\Omega} \subseteq \Omega$ then

$$
\Psi_{\bar{\Omega}}\left(A_{i} x\right) \geq \Psi_{\bar{\Omega}}(x), \quad \forall i \in \mathbb{N}_{q},
$$

for all $x \in \mathbb{R}^{n}$.

Proof: The set $\bar{\Omega}$ is a $\mathrm{C}^{*}$-set from the proof of Proposition 1. Consider first $x \in \partial \bar{\Omega}$, which implies $\Psi_{\bar{\Omega}}(x)=1$. From the definition of $\bar{\Omega}$, in (15), for every $i \in \mathbb{N}_{q}$ either $x \in \partial\left(A_{i}^{-1} \Omega\right)$ or $x \notin A_{i}^{-1} \Omega$. In fact, otherwise, there would be $i(x) \in \mathbb{N}_{q}$ such that $x \in \operatorname{int}\left(A_{i(x)}^{-1} \Omega\right) \subseteq \operatorname{int}(\bar{\Omega})$, which contradicts $x \in \partial \bar{\Omega}$. For every $i \in \mathbb{N}_{q}$ such that $x \in \partial\left(A_{i}^{-1} \Omega\right)$ one have that $A_{i} x \in \partial \Omega$, while if $i \in \mathbb{N}_{q}$ is such that $x \notin A_{i}^{-1} \Omega$ then $A_{i} x \notin \Omega$. Thus it follows that $A_{i} x \notin \operatorname{int}(\Omega)$ for all $i \in \mathbb{N}_{q}$, and then, from $\bar{\Omega} \subseteq \Omega$, also $A_{i} x \notin \operatorname{int}(\bar{\Omega})$, for all $i \in \mathbb{N}_{q}$. Since $\Psi_{\bar{\Omega}}(y) \geq 1$ if and only if $y \notin \operatorname{int}(\bar{\Omega})$, then condition (16) holds at $x \in \partial \bar{\Omega}$. Finally, since every $x \in \mathbb{R}^{n}$ is on the boundary of a scalar multiple of $\bar{\Omega}$ and from the homogeneity of the Minkowski function of a $C^{*}$-set, condition (16) is satisfied for every $x \in \mathbb{R}^{n}$.

Proposition 3 states that the existence of a $C^{*}$-set $\Omega$ such that $\bar{\Omega}$, defined in (15), is contained in $\Omega$ suffices to prove non-stabilizability. Notice that $\bar{\Omega}$ is the union of the preimages of $\Omega$ through $A_{i}$ for all $i \in \mathbb{N}_{q}$. In case the $\mathrm{C}^{*}$-set is such that $\bar{\Omega} \subseteq \operatorname{int}(\Omega)$, the Minkowski function increases at any point of the state space, for every mode.

Corollary 2 Given the switched system (1) and the $C^{*}$-sets $\Omega \subseteq \mathbb{R}^{n}$ and $\bar{\Omega} \subseteq \mathbb{R}^{n}$ as in (15), if $\bar{\Omega} \subseteq \operatorname{int}(\Omega)$ then

$$
\Psi_{\bar{\Omega}}\left(A_{i} x\right)>\Psi_{\bar{\Omega}}(x), \quad \forall i \in \mathbb{N}_{q},
$$

for all $x \in \mathbb{R}^{n}$.

Hence, the existence of a $C^{*}$-set $\Omega \subseteq \mathbb{R}^{n}$ such that $\bar{\Omega}$ as in (15) satisfies $\bar{\Omega} \subseteq \Omega$ implies that there is not a stabilizing switching sequence. We introduce an algorithm whose finite termination is related to the existence of such a set and then to the non-stabilizability of the switched linear system (1).

Algorithm 3 Test of non-stabilizability for the system (1) such that Assumption 1 holds.

- Initialization: given the $C^{*}$-set $\Omega \subseteq \mathbb{R}^{n}$, define $\Omega_{0}=\hat{\Omega}_{0}=$ $\Omega$ and $k=0$;

- Iteration for $k \geq 0$ compute $\Omega_{k+1}^{i}$ and $\Omega_{k+1}$ as in (3) and define

$$
\hat{\Omega}_{k+1}=\left(\bigcup_{j \in \mathbb{N}_{k+1}} \Omega_{j}\right) \cup \Omega .
$$

- Stop if $\Omega_{k+1} \subseteq \hat{\Omega}_{k}$; denote $\hat{N}=k$ and $\hat{\Omega}=\hat{\Omega}_{\hat{N}}$ : the system (1) is non-stabilizable.

The finite termination of Algorithm 3 is a sufficient condition for non-stabilizability of the system (1), as proved below.

Theorem 4 If Algorithm 3 ends with finite $\hat{N}$ then there is no switching law stabilizing the switched system (1).

Proof: For every $k \in \mathbb{N}$, the set in (18) is a $\mathrm{C}^{*}$-set from Proposition 1 and the fact that the union of $\mathrm{C}^{*}$-sets is a $\mathrm{C}^{*}$ set. Suppose that Algorithm 3 ends with finite $\hat{N}$ and define $\bar{\Omega}=\bigcup_{i \in \mathbb{N}_{q}} A_{i}^{-1} \hat{\Omega}$. For all nonsingular matrix $A \in \mathbb{R}^{n \times n}$ and every $\mathrm{C}^{*}$-sets $D, E \subseteq \mathbb{R}^{n}$ we have that also the set $A^{-1}(D \cup$ $E)=\left(A^{-1} D\right) \cup\left(A^{-1} E\right)$ is a $C^{*}$-set. It follows that

$$
\begin{aligned}
\bar{\Omega} & =\bigcup_{i \in \mathbb{N}_{q}} A_{i}^{-1}\left(\left(\bigcup_{j \in \mathbb{N}_{\hat{N}}} \Omega_{j}\right) \cup \Omega\right)= \\
& =\Omega_{\hat{N}+1} \cup\left(\bigcup_{j \in \mathbb{N}_{\hat{N}}} \Omega_{j}\right) \subseteq\left(\bigcup_{j \in \mathbb{N}_{\hat{N}}} \Omega_{j}\right) \cup \Omega=\hat{\Omega} .
\end{aligned}
$$

Then from Proposition 3, the Minkowski function of $\bar{\Omega}$ is non-decreasing for every mode and at every point of the state space and the system is non-stabilizable.

Remark 7 Notice that the termination condition of Algorithm 3 is equivalent to $\hat{\Omega}_{k+1} \subseteq \hat{\Omega}_{k}$. In fact, we have that

$$
\hat{\Omega}_{k+1}=\Omega_{k+1} \cup\left(\bigcup_{j \in \mathbb{N}_{k}} \Omega_{j}\right) \cup \Omega=\Omega_{k+1} \cup \hat{\Omega}_{k} \subseteq \hat{\Omega}_{k},
$$

which holds if and only if $\Omega_{k+1} \subseteq \hat{\Omega}_{k}$ and $\hat{\Omega}_{k} \subseteq \hat{\Omega}_{k}$, trivially satisfied the latter.

A class of switched systems such that finite termination is assured is characterized in the following theorem.

Theorem 5 If there is a continuous positive definite and radially unbounded function $\bar{V}: \mathbb{R}^{n} \rightarrow \mathbb{R}$ such that

$$
\bar{V}\left(A_{i} x\right)>\bar{V}(x), \quad \forall i \in \mathbb{N}_{q},
$$

for all $x \in \mathbb{R}^{n}$, then Algorithm 3 ends with finite $\hat{N}$ and the switched system (1) is non-stabilizable. 
Proof: From (19) and Assumption 1, it follows that

$$
\bar{V}\left(A_{i}^{-1} x\right)<\bar{V}(x), \quad \forall i \in \mathbb{N}_{q}
$$

for all $x \in \mathbb{R}^{n}$. Consider the set defined

$$
\bar{\Omega}^{\bar{V}}=\left\{x \in \mathbb{R}^{n}: \bar{V}(x) \leq 1\right\},
$$

which is compact and such that $0 \in \operatorname{int}\left(\bar{\Omega}^{\bar{V}}\right)$, being $\bar{V}$ continuous, positive definite and radially unbounded, as the set $\Omega^{V}$ in (10). Then the $C^{*}$-set $\Omega$ initializing Algorithm 3 can be chosen such that $\Omega \subseteq \bar{\Omega}^{\bar{V}}$, after an adequate scaling. From (20), we have that there is a finite $N \in \mathbb{N}$ such that

$$
\prod_{k \in \mathbb{N}_{N}} A_{i(k)}^{-1} x \in \Omega, \quad \forall x \in \bar{\Omega}^{\bar{V}},
$$

and every sequence of modes $i(k) \in \mathbb{N}_{q}$ with $k \in \mathbb{N}_{N}$, which implies $\Omega_{N} \subseteq \Omega$, being $\Omega \subseteq \bar{\Omega}^{\bar{V}}$. Then, since $\Omega \subseteq \hat{\Omega}_{N}$, see (18), Algorithm 3 stops after $N$ iterations at most.

In the case that the function $V(\cdot)$ is such that $\bar{V}\left(A_{i} x\right) \geq$ $\bar{V}(x)$, for all $i \in \mathbb{N}_{q}$, the finite termination of Algorithm 3 is not ensured (nor excluded) by Theorem 5. In particular, if stability is ensured by a function $V(\cdot)$ such that $\bar{V}\left(A_{i} x\right)=$ $\bar{V}(x)$, for all $i \in \mathbb{N}_{q}$ and every $x \in \mathbb{R}^{n}$, that does not guarantee convergence, the algorithm could terminate in finite time or not, as shown in the following illustrative example.

Example 1 Consider the linear system given by (1) with $q=1$ and $A_{1}=R(\beta \pi)$, where $R(\theta)$ is the rotation matrix of angle $\theta$ and with $\beta \in \mathbb{R} \backslash \mathbb{Q}$ such that $\beta \in(0,0.5)$. The system is stable but not convergent to the origin, then a Lyapunov function does not exist and Algorithm 1 has not a finite termination. Suppose that Algorithm 3 is applied with initial set $\Omega=\left\{x \in \mathbb{R}^{2}:\|x\|_{\infty} \leq 1\right\}$, which is a square centered in the origin. The sequence of sets $\Omega_{k}$ with $k \in \mathbb{N}$ are the square $\Omega$ rotated of the angle $k \beta \pi$ with $k \in \mathbb{N}$. We prove that for every $m, k \in \mathbb{N}$ with $k>m$, the sets $\Omega_{k} \neq \Omega_{m}$. In fact otherwise we would have that the rotation is the same, except for a multiple of $\pi / 2$, since the square does not change only if it is rotated of $d \pi / 2$ with $d \in \mathbb{N}$. But this implies that, for an adequate $d \in \mathbb{N}$, we have $k \beta \pi=m \beta \pi+d \pi / 2$ and then $\beta=d / 2(k-m)$, which contradicts the fact that $\beta$ is an irrational number. Hence Algorithm 3 does not end in finite time, since $\hat{\Omega}_{k}$ is the union of $\Omega_{j}$ with $j<k$. Conversely, choosing the euclidean norm ball as initial condition, i.e. $\Omega=\mathbb{B}^{2}$, the algorithm stops at the first step. In fact, we have $\Omega_{1}=\hat{\Omega}_{0}=\Omega$. Notice that the euclidean ball is the only $\mathrm{C}^{*}$-set whose Minkowski function does not increase (nor decrease) along the trajectories. Furthermore, the sequence $\hat{\Omega}_{k}$ generated by Algorithm 3 initialized with the square converges to the euclidean ball of radius $\sqrt{2}$ for $k \rightarrow \infty$.

The finite termination of Algorithm 3 is only a sufficient condition for non-stabilizability of the system (1), see Theorem 4 . The finite termination is attained if and only if there
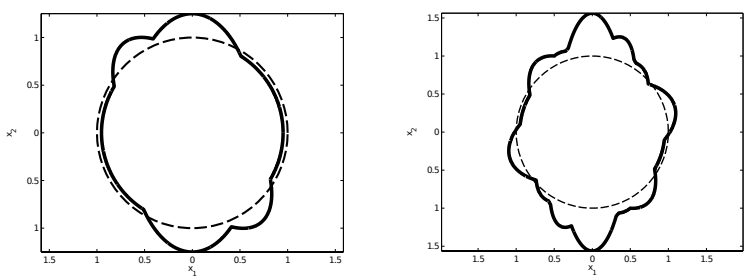

Fig. 1. Ball $\mathbb{B}^{2}$ in dashed and $\Omega_{1}$ and $\bigcup_{k \in \mathbb{N}_{2}} \Omega_{k}$ solid line.

is a positive definite radially unbounded function increasing along the trajectories, for every possible switching sequence, see Theorem 5. There are cases in which the system (1) is non-stabilizable but Algorithm 3 does not ends in finite time (and Algorithm 1 neither, clearly).

\section{Lyapunov-Metzler approach}

Conditions for the stabilizability of switching system are available in literature. Those based on the Lyapunov-Metzler inequalities are among the less conservative. Such conditions are given by nonlinear matrix inequalities whose solution is often numerically intractable, see $[11,10]$. In the continuous-time context, it has been proved that the existence of a stable convex combination of the matrices $A_{i}$, with $i \in \mathbb{N}_{q}$, is a sufficient condition (and also necessary for $q=2$ ) for the Lyapunov-Metzler inequalities to hold. Such a condition on the convex combinations of the modes is at the core of the stabilizing techniques in the literature, see [18]. For discrete-time systems, such a relation is less evident and other available sufficient conditions are more conservative, see [11]. It is worth then to directly consider the Lyapunov-Metzler condition to obtain an appropriate comparison, although its evaluation would require a computationally demanding approach. It has to be recalled, nevertheless, that Lyapunov-Metzler conditions deal with nonconvex Lyapunov functions, namely homogeneous ones whose level sets are union of ellipsoids.

\section{Numerical examples}

Example 2 Consider the system (1) with $q=4, n=2$ and

$$
\begin{aligned}
& A_{1}=\left[\begin{array}{cc}
1.5 & 0 \\
0 & -0.8
\end{array}\right], \quad A_{2}=1.1 R\left(\frac{2 \pi}{5}\right) \\
& A_{3}=1.05 R\left(\frac{2 \pi}{5}-1\right), \quad A_{4}=\left[\begin{array}{cc}
-1.2 & 0 \\
1 & 1.3
\end{array}\right] \text {. }
\end{aligned}
$$

The matrices $A_{i}$, with $i \in \mathbb{N}_{4}$, are not Schur, which implies that the system (1) is not stabilizable by any constant switching law. We apply Algorithm 1 with $\Omega=\mathbb{B}^{2}$. The sets $\Omega_{k}$, $k \in \mathbb{N}$ are thus unions of ellipsoids. The result at the first step is depicted in Figure 1, left. $\Omega_{1}$ is the union the four ellipsoids $A_{j}^{-1} \mathbb{B}^{2}$, with $j \in \mathbb{N}_{4}$. It is clear that $\mathbb{B}^{2}$ does not belong 


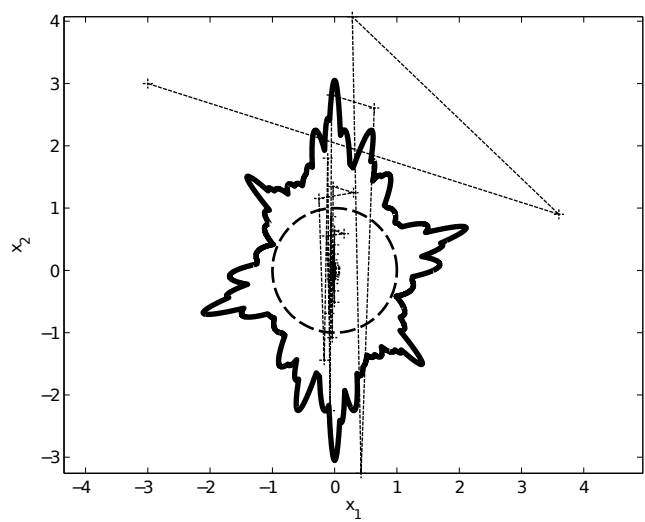

Fig. 2. Ball $\mathbb{B}^{2}$ in dashed and $\bigcup_{k \in \mathbb{N}_{5}} \Omega_{k}$ in solid line. Trajectory starting from $x_{0}=(-3,3)^{T}$ in dotted line.

to $\Omega_{1}$. The next step of the algorithm leads to a set $\bigcup_{k \in \mathbb{N}_{2}} \Omega_{k}$ given by the union of the $4+4^{2}=20$ ellipsoids at most, i.e. $A_{j}^{-1} \mathbb{B}^{2}$ with $j \in \mathbb{N}_{4}$ and $A_{j}^{-1} A_{i}^{-1} \mathbb{B}^{2}$, for all $(i, j) \in \mathbb{N}_{4} \times \mathbb{N}_{4}$. Since $\mathbb{B}^{2}$ does not belong to $\bigcup_{k \in \mathbb{N}_{2}} \Omega_{k}$, see Figure 1 right, the termination condition is not satisfied. The algorithm stops at the fifth iteration. Figure 2 emphasizes that $\mathbb{B}^{2}$ is included in $\bigcup_{k \in \mathbb{N}_{5}} \Omega_{k}$. A stabilizing switching law, satisfying (13) is given in Figure 3 for the initial condition $x_{0}=(-3,3)^{T}$. The Lyapunov function converges to zero, see Figure 3.
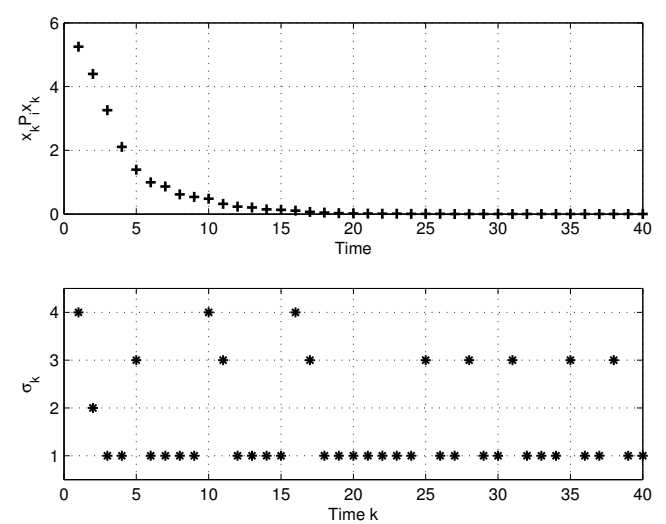

Fig. 3. Lyapunov function and switching control law in time.

Example 3 As a second example, consider for $q=n=2$ :

$$
A_{1}=\left[\begin{array}{cc}
0 & -1.01 \\
1 & -1
\end{array}\right], \quad A_{2}=\left[\begin{array}{cc}
0 & -1.01 \\
1 & -0.5
\end{array}\right] \text {. }
$$

The Lyapunov-Metzler approach results in a set of linear matrix inequalities once two parameters are fixed, both contained in $[0,1]$, see [11]. The LMI conditions have been checked on a grid of these parameters, with step of 0.01 . No admissible solution was found, whereas our algorithm stops at the third step. Figure 4 shows that $\mathbb{B}^{2} \subseteq \operatorname{int}\left(\bigcup_{k \in \mathbb{N}_{3}} \Omega_{k}\right)$. The Lyapunov function and the switching law are given in Figure 5. Notice that the Lyapunov function is not a decreasing function, but only a non-increasing one which is strictly decreasing at least once every three (the number of steps of the algorithm) instants, as proved in the main result.

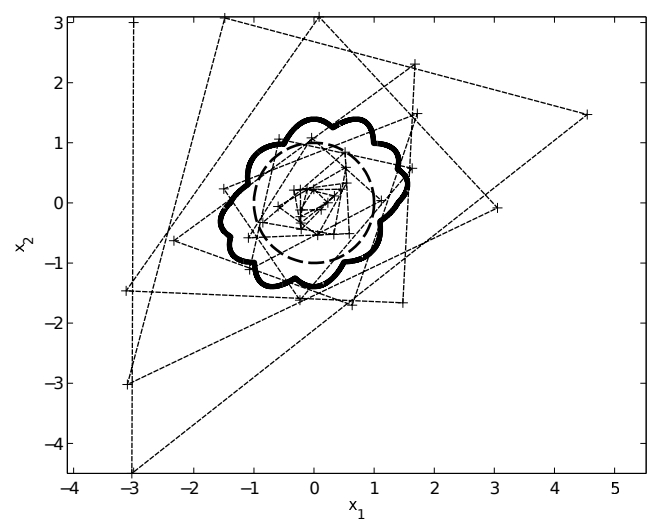

Fig. 4. Ball $\mathbb{B}^{2}$ in dashed and $\bigcup_{k \in \mathbb{N}_{3}} \Omega_{k}$ in solid line. Trajectory starting from $x_{0}=(-3,3)^{T}$ in dotted line.
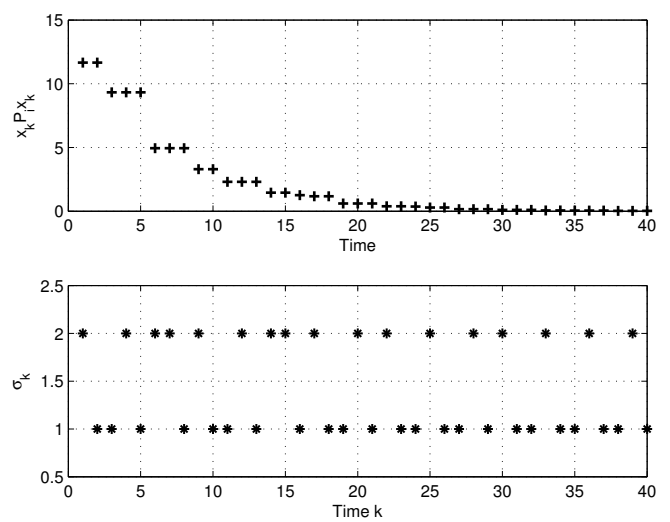

Fig. 5. Lyapunov function and switching control law in time.

Example 4 As mentioned above, there are undecidable cases for which neither Algorithm 1 nor Algorithm 3 are finite. Consider

$$
A_{1}=\left[\begin{array}{cc}
1.3 & 0 \\
0 & 0.9
\end{array}\right] R(\theta), \quad A_{2}=\left[\begin{array}{cc}
1.4 & 0 \\
0 & 0.8
\end{array}\right],
$$

with $\theta=0$ for the moment. Matrices $A_{1}$ and $A_{2}$ are not Schur. Four steps are applied for each algorithm. The results are drawn in Figure 6 . Notice that $x=(1,0)^{T}$ is related to an unstable eigenvalue for both modes, then Algorithm 1 cannot be finite. Moreover in direction $x=(0,1)^{T}, \cup_{k \in \mathbb{N}_{i}} \Omega_{k}$ is increasing with $i$, then Algorithm 3 cannot be finite. 


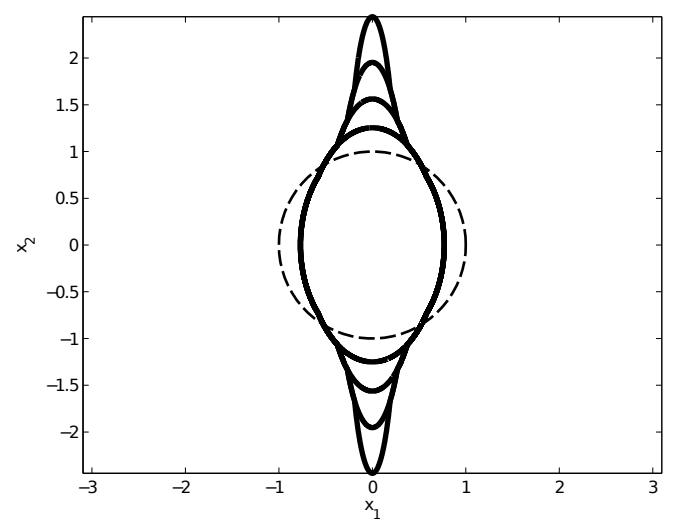

Fig. 6. Ball $\mathbb{B}^{2}$ in dashed and $\cup_{k \in \mathbb{N}_{i}} \Omega_{k}$, for $i \in \mathbb{N}_{4}$ in solid line.

Example 5 Consider the system given by (22) with $\theta=\frac{\pi}{5}$, which means that the first mode performs now a rotation of $\frac{\pi}{5}$. Algorithm 1 stops after seven steps implying the stabilizability of the system. The result is given in Figure 7.

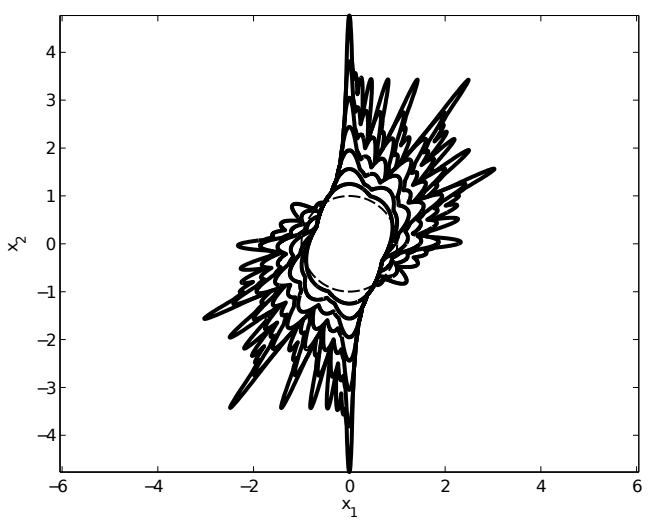

Fig. 7. Ball $\mathbb{B}^{2}$ in dashed and $\cup_{k \in \mathbb{N}_{i}} \Omega_{k}$, for $i \in \mathbb{N}_{7}$ in solid line.

Example 6 An example, leading to the finiteness of Algorithm 3 , is now proposed. We consider

$$
A_{1}=2\left[\begin{array}{cc}
0 & -1.01 \\
1 & -1
\end{array}\right], \quad A_{2}=2\left[\begin{array}{cc}
0 & -1.01 \\
1 & -0.5
\end{array}\right] .
$$

The stop criterion is attained for Algorithm 3 in only one step, as shown in Figure 8, then the system is not stabilizable.

Example 7 Consider $q=2$ and $n=3$ with

$$
A_{1}=\left[\begin{array}{ccc}
1.2 & 0 & 0 \\
-1 & 0.8 & 0 \\
0 & 0 & 0.5
\end{array}\right], \quad A_{2}=\left[\begin{array}{ccc}
0.7 & 0 & 0 \\
0 & -0.6 & -2 \\
0 & 0 & -1.2
\end{array}\right] \text {. }
$$

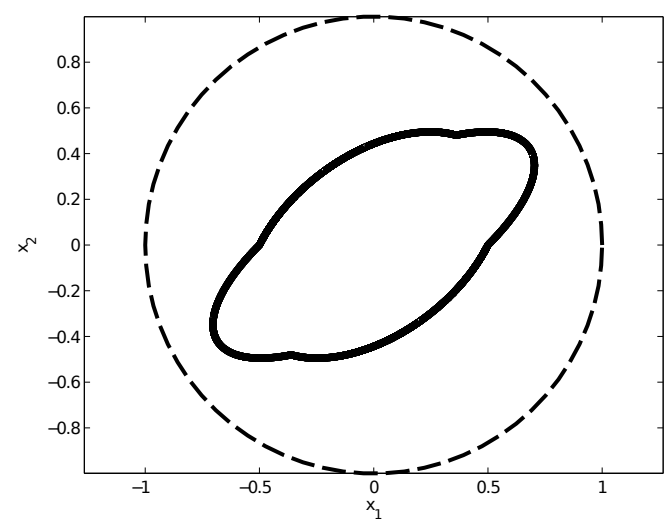

Fig. 8. Ball $\mathbb{B}^{2}$ in dashed and $\cup_{k \in \mathbb{N}_{1}} \Omega_{k}$ in solid line.

$A_{1}$ and $A_{2}$ are not Schur. The ball $\mathbb{B}^{3}$ is chosen as initial set. Algorithm 1 ends at the third step. The Figure 9 depicts the ball $\mathbb{B}^{3}$ and the sets $\cup_{k \in \mathbb{N}_{j}} \Omega_{k}$, with $j \in \mathbb{N}_{3}$.

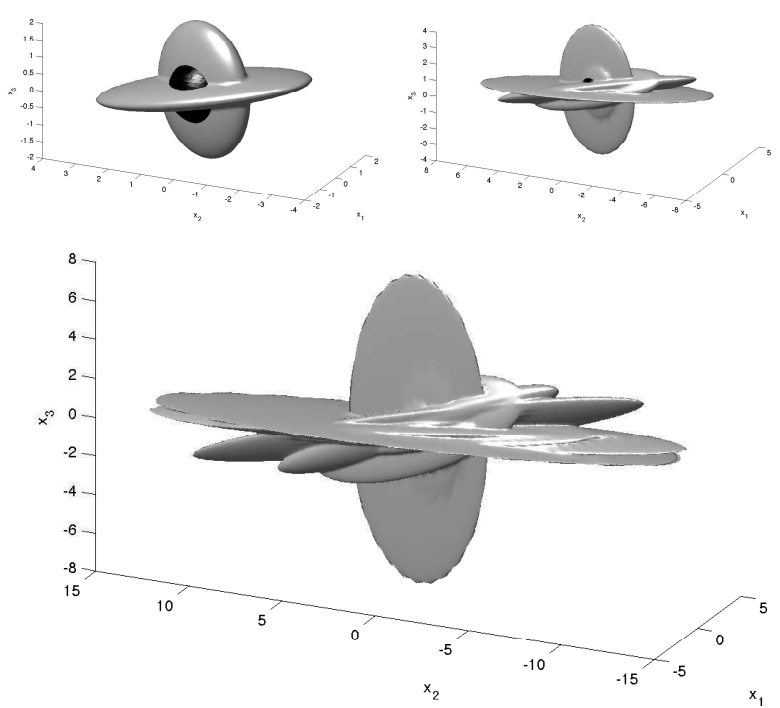

Fig. 9. Ball $\mathbb{B}^{3}$ in dark and $\cup_{k \in \mathbb{N}_{j}} \Omega_{k}$, for $j \in \mathbb{N}_{3}$, in light gray.

\section{Acknowledgements}

This work was partially supported by ANR project ArHyCo, Programme "Systèmes Embarqués et Grandes Infrastructures" - ARPEGE, contract number ANR-2008 SEGI 004 01- 30011459. This research has received funding from the European Community's Seventh Framework Programme (FP7/2007-2013) under grant agreement $n^{\circ}$ 257462: HYCON2 Network of Excellence "Highly-Complex and Networked Control Systems". The authors would like to thank Professor Jamal Daafouz for the helpful discussions. 


\section{Conclusions}

The issue of the stabilizability of discrete-time linear switched systems has been studied in this paper. Via a set-theory approach, necessary and sufficient conditions for the stabilizability and sufficient ones for non-stabilizability have been provided. The family of nonconvex, homogeneous functions induced by a $\mathrm{C}^{*}$-set is proved to be a class of universal Lyapunov functions for switched systems. In addition, the stabilizing switching laws are characterized. Our method allows moreover to stabilize counter-examples for the Lyapunov-Metzler approach. Several academic illustrations are proposed to strengthen the discussions and to emphasize the efficiency of our approach.

\section{References}

[1] P. H. Bauer, K. Premaratne, and J. Durán. A necessary and sufficient condition for robust asymptotic stability of time-variant discrete systems. IEEE Transactions on Automatic Control, 38(9):1427-1430, 1993.

[2] D. P. Bertsekas. Infinite-time reachability of statespace regions by using feedback control. IEEE Transactions on Automatic Control, 17:604-613, 1972.

[3] F. Blanchini. Ultimate boundedness control for discrete-time uncertain systems via set-induced Lyapunov functions. IEEE Transactions on Automatic Control, 39:428-433, 1994.

[4] F. Blanchini. Nonquadratic Lyapunov functions for robust control. Automatica, 31:451-461, 1995.

[5] F. Blanchini. Set invariance in control. Automatica, 35:1747-1767, 1999.

[6] F. Blanchini and S. Miani. Set-Theoretic Methods in Control. Birkhäuser, 2008.

[7] F. Blanchini and C. Savorgnan. Stabilizability of switched linear systems does not imply the existence of convex Lyapunov functions. Automatica, 44:11661170, 2008.

[8] U. Boscain. Stability of planar switched systems: the linear single input case. SIAM J. Control Optim., 41(1):89-112, 2002.

[9] M. Fiacchini and M. Jungers. Necessary and sufficient condition for stabilizability of discrete-time linear switched systems: a set-theory approach. In Proceedings of the IFAC Joint conference, Symposium System Structure and Control, 2013.

[10] J. C. Geromel and P. Colaneri. Stability and stabilization of continuous-time switched linear systems. SIAM J. Control Optim., 45(5):1915-1930, 2006.

[11] J. C. Geromel and P. Colaneri. Stability and stabilization of discrete-time switched systems. International Journal of Control, 79(7):719-728, July 2006.

[12] E. G. Gilbert and K. Tan. Linear systems with state and control constraints: The theory and application of maximal output admissible sets. IEEE Transactions on Automatic Control, 36:1008-1020, 1991.

[13] L. Gurvits, R. Shorten, and O. Mason. On the stability of switched positive linear systems. IEEE Transactions on Automatic Control, 52(6):1099-1103, 2007.

[14] R. M. Jungers. The Joint Spectral Radius: Theory and Applications. Springer-Verlag., Berlin Heidelberg, June 2009.

[15] I. Kolmanovsky and E. G. Gilbert. Theory and computation of disturbance invariant sets for discrete-time linear systems. Mathematical Problems in Engineering, 4:317-367, 1998.

[16] A. Kruszewski, R. Bourdais, and W. Perruquetti. Converging algorithm for a class of BMI applied on statedependent stabilization of switched systems. Nonlinear Analysis: Hybrid Systems, 5:647-654, 2011.

[17] J. W. Lee and G. E. Dullerud. Uniformly stabilizing sets of switching sequences for switched linear systems. IEEE Transactions on Automatic Control, 52:868-874, 2007.

[18] D. Liberzon. Switching in Systems and Control. Birkhäuser, Boston, MA, 2003.

[19] D. Liberzon and A. S. Morse. Basic problems in stability and design of switched systems. IEEE Control Systems Magazine, 19:59-70, 1999.

[20] H. Lin and P. J. Antsaklis. Synthesis of uniformly ultimate boundedness switching laws for discrete-time uncertain switched linear systems. In Proceedings of the 42nd IEEE Conference on Decision and Control, pages 4806-4811, 2003.

[21] H. Lin and P. J. Antsaklis. Persistent disturbance attenuation properties for networked control systems. In Proceedings of the 43rd IEEE Conference on Decision and Control, pages 953-958, 2004.

[22] H. Lin and P. J. Antsaklis. Stability and stabilizability of switched linear systems: a survey of recent results. IEEE Transaction on Automatic Control, 54(2):308$322,2009$.

[23] J. Lunze and F. Lamnabhi-Lagarrigue, editors. Handbook of Hybrid Systems Control: Theory, Tools, Applications. Cambridge, 2009.

[24] A. P. Molchanov and Y. S. Pyatnitskiy. Criteria of asymptotic stability of differential and difference inclusions encounterd in control theory. Systems \& Control Letters, 13:59-64, 1989.

[25] R. T. Rockafellar. Convex Analysis. Princeton University Press, USA, 1970.

[26] R. Schneider. Convex bodies: The Brunn-Minkowski theory. Cambridge University Press, Cambridge, England, 1993.

[27] Z. Sun and S. S. Ge. Stability Theory of Switched Dynamical Systems. Springer, 2011.

[28] M. A. Wicks, P. Peleties, and R. A. De Carlo. Construction of piecewise Lyapunov functions for stabilizing switched systems. In Proceedings of the 33rd IEEE Conference on Decision and Control, pages 34923497, 1994. 